\title{
Image-Guided Development of Biomaterials: Enabling Technologies Shaping and Expediting the Future of Materials in Medicine
}

The demand for biomaterial-based technologies is increasing at a rapid pace, especially in light of the aging worldwide population. As biomaterials evolve from purely replacement parts to more sophisticated, multifaceted systems incorporating various biological, chemical and mechanical components, the cost and time required to complete the product development pipeline is also sharply rising. A single FDA approval for a drug and/or biologic can be to the tune of over 10 years with costs into the hundreds of millions of dollars. The high cost is due to lengthy and often disappointing preclinical studies looking at device or drug formulation, efficacy and safety. Only $0.05-0.1 \%$ of therapeutic candidates actually move on to FDA trials, which have a $25-35 \%$ failure rate at each step. Even after a new entity makes it to the market, device failure rates are surprisingly high - for example in 2009, there were nearly 600 reports of device failure or complication at the FDA. ${ }^{1}$ This staggering funnel in the approval pipeline highlights many shortcomings of preclinical work. Importantly, a lack of nondestructive processes and tools that enable accurate prediction of device success and safety temporally, while minimizing the likelihood of unexpected negative side effects at the clinical level, considerably increases the costs and time of laboratory and preclinical trials. A recent report in Science Translational Medicine articulates this challenge: "Careful evaluation of the necessary parameters for success can help minimize the chances of unexpected negative off-target effects in clinical trials, reduce the regulatory pathway, and increase the commercial desirability and profitability of the therapy as it transitions from laboratory to clinic". ${ }^{2}$

The scientific community relies on well-established studies and models to carry out preclinical testing, but these tests may not be ideal or even relevant for the increasingly complex devices comprised of innovative biomaterials. In vivo animal work is expensive, time consuming, cumbersome (as current strategies are typically combined with ex vivo analysis), and ethically questioned in many circles. In vitro and mathematical models of in vivo systems are potentially intriguing, but it is unlikely that any one such system can account for the multitude of complex processes taking place at any given time in vivo. Devices such as 'lab-on-a chip' technology have the potential of integrating a number of diagnostic tests, but their application in testing larger biomaterial constructs is limited. It thus stands to reason that the streamlined, efficient evaluation of the material or medical device performance in vivo is now more than ever of paramount importance to the advancement of the biomaterials field. A rethinking of the tools that are currently utilized for analysis of most materials and the implantable devices made from them may be the key to progress.

The purpose of this special issue is to showcase the innovative and

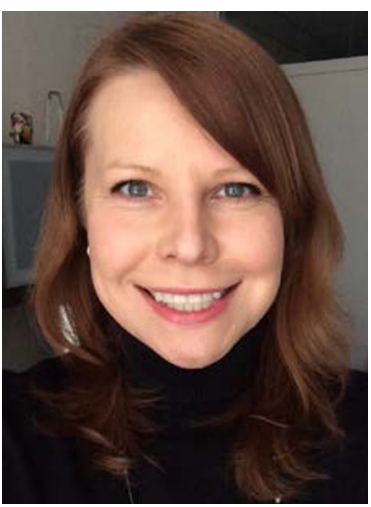

Agata A. Exner

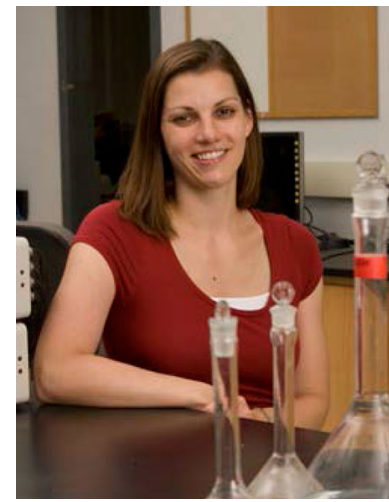

Rebecca Kuntz Willits broadly-enabling imaging tools that are currently under development and that stand to revolutionize the advancement and translation of new biomaterials in the very near future. We have gathered researchers to provide examples of primary research and review papers highlighting technologies in the areas of ultrasound, MRI, and fluorescence as well as tomography techniques which are now frequently utilized for nondestructive biomaterial characterization for drug delivery and tissue engineering approaches. Key to this special edition is the unique and strong cross disciplinary collaborations that have been developed to further these non-destructive techniques.

For this special edition, we first present review papers that detail nondestructive techniques and their broad applications. Ultrasound, photoacoustic microscopy, mesoscopic fluorescence molecular tomogra- 
phy, and near infrared spectroscopy are all well described by leaders in the fields of imaging and materials. These reviews detail the various utilizations of the technologies from nondestructive to noninvasive evaluation of engineered tissues and biomaterials. Next, we have assembled a group of review papers that investigate nondestructive techniques for particular responses, such as evaluation of immune response, biomechanics, tissue metabolism, and cartilage. The obvious need for improved monitoring and imaging agents is also highlighted. These papers highlight the innovative methods researchers have found to better characterize cells and tissues nondestructively. Finally, we have compiled a set of original papers that seek to apply nondestructive techniques for imaging and analysis of biomaterials and hydrogels, tissue engineered constructs, and neural stem cell functionality.

As demonstrated by the following research reports and reviews, by using imaging to noninvasively study biomaterials at their intended site of implantation over time, the relevant data needed to accurately predict patient-associated risks from a technology can be collected quickly and easily. The resulting imaging toolbox can enable scientists to balance practicality with innovation and accelerate the preclinical investigation process by increasing by many orders of magnitude the amount of information/data collected from each experiment. For example, one can envision that in the future the separate destructive experiments to examine rates of drug release, implant morphology, tissue response to the implant, mechanical properties, and tissue response at bulk and molecular levels, can easily be replaced by one multi-modality imaging session of one implant in one animal. The gain in information and reduction in cost, time and effort of this process can be immense. We hope that you find this special edition useful in your scientific quests.

\section{REFERENCES}

${ }^{1}$ Bryers, J. D., C. M. Giachelli, and B. D. Ratner. Engineering biomaterials to integrate and heal: the biocompatibility paradigm shifts. Biotechnol. Bioeng. 109:1898-1911, 2012.

${ }^{2}$ Pashuck, E. T., and M. M. Stevens. Designing regenerative biomaterial therapies for the clinic. Sci. Transl. Med. 4:160sr164, 2012.

Agata A. Exner

Department of Radiology, Case Center for Imaging Research, Case Western Reserve University, Cleveland, $\mathrm{OH}$ 44106, USA

Electronic mail: aas11@case.edu

Rebecca Kuntz Willits

Department of Biomedical Engineering, The University of Akron, Akron, OH 44325-0302, USA 